\title{
The Neglected Cousin of the Hepatocyte: How Gallbladder Epithelial Cells Might Contribute to Cholesterol Gallstone Formation
}

\author{
Arne Dikkers · Uwe J. F. Tietge
}

Received: 10 December 2012/Accepted: 19 December 2012/Published online: 31 January 2013

(C) Springer Science+Business Media New York 2013

The regulation of biliary cholesterol secretion by hepatocytes has received much interest [1], since it has a major impact on two highly relevant disease complexes, namely, the generation of cholesterol gallstones which have an overall prevalence of 10-20\% in developed countries [2] and reverse cholesterol transport [3], an important protective pathway against atherosclerotic cardiovascular disease as well as the main cause for morbidity and mortality in industrialized societies [4]. Less attention has thus far been given to the potential participation of the gallbladder itself in metabolism, which is somewhat surprising, since the gallbladder has the capacity to actively absorb cholesterol from bile and thereby modulate bile cholesterol content [5]. Changes in transporters mediating this metabolic activity of gallbladder epithelial cells could conceivably contribute to cholesterol gallstone formation in disease. However, only a very limited number of studies exist that have functionally explored the relative contributions of known cholesterol transport proteins to cholesterol transport in and out of the gallbladder (Fig. 1).

The study by Yoon et al. [6] in this issue of Digestive Diseases and Sciences reports that the ATP-binding cassette transporters G5/G8 (ABCG5/G8) are expressed on the apical side, while ABCA1 is found on the basolateral side of gallbladder epithelial cells. Both transporters are upregulated in gallbladders from patients with cholesterol gallstone disease [6]. Thus far, on the apical side the expression of ACBG5/G8 [7], scavenger receptor class B

\footnotetext{
A. Dikkers · U. J. F. Tietge ( $)$

Department of Pediatrics, University Medical Center Groningen,

University of Groningen, Hanzeplein 1, 9713 GZ Groningen,

The Netherlands

e-mail: u_tietge@yahoo.com
}

type I (SR-BI) [8], cubilin and megalin [9] was reported in gallbladder epithelial cells. The obligate heterodimer ABCG5/G8 is a cholesterol transporter expressed in enterocytes and hepatocytes, where it mediates the excretion of cholesterol and plant sterols out of the cell [1]. About $70 \%$ of total biliary cholesterol secretion is ABCG5/G8 dependent. Total genetic deletion of ABCG5/ G8 produces sitosterolemia, a disease associated with hyperabsorption of cholesterol and plant sterols that is linked to accelerated atherosclerosis [1]. Interestingly, the ABCG5/G8 locus has also long been implicated in the formation of cholesterol gallstones [10]. Recently, specific coding variants have been identified in genome-wide association studies that likely result in a gain-of-function of ABCG5/G8, supposedly translating into increased biliary cholesterol secretion [11]. Neither in animal experiments nor in human material has the functionality of ABCG5/G8, specifically in the gallbladder, been explored; although, derived from their function in liver and intestine increasing cholesterol secretion into bile would be expected. In this context the higher expression of these transporters noted by Yoon et al. [6] in patients with gallstone disease points towards a causative contribution of ABCG5/G8 to gallstone formation. However, results from studies using the perfused gallbladder model indicated reduced cholesterol absorption from bile in gallbladders from gallstone disease patients [12], although the methods used did not formally distinguish between decreased uptake versus uptake followed by accelerated resecretion, e.g. via increased ABCG5/G8 expression [12]. Moreover, decreased expression of uptake receptors on the apical side such as cubilin or megalin might also explain these results. Interestingly, cholesterol uptake and secretion can occur on the apical side [13] providing further indirect evidence for a potential functional role of $\mathrm{ABCG} 5 / \mathrm{G} 8$ in the gallbladder. 


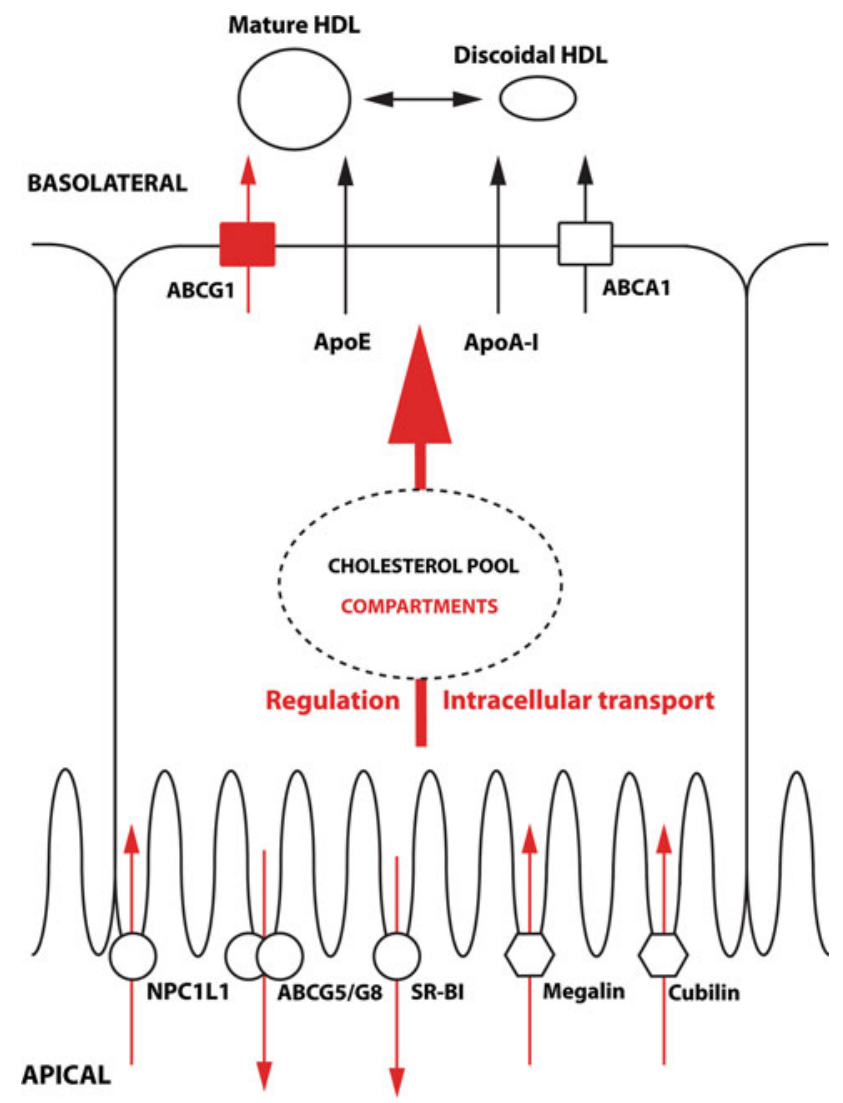

Fig. 1 Cholesterol metabolism in the gallbladder epithelial cell. Transport proteins are visualized as symbols, metabolic fluxes as arrows. Known proteins and pathways are shown in black, while as yet unidentified or hypothetical components of cholesterol metabolism in these cells are shown in red. Further details are provided in the text

SR-BI, mainly known as the selective uptake receptor for HDL cholesterol [4], has also been detected on the apical membrane of gallbladder epithelial cells [8]. However, although in this case functional studies were carried out, no clear role could be assigned to the expression of SR-BI in the context of cholesterol handling by gallbladder epithelial cells [8]. Correlation of transporter expression levels with actual cholesterol transport in clinical material would be worthwhile.

Nieman-Pick C 1-like protein 1 (NPC1L1) is another potential apical cholesterol transporter expressed in enterocytes, hepatocytes and gallbladder in humans [1]. The molecular target of ezetimibe, NPC1L1, mediates cholesterol uptake although its functional impact has thus far not been investigated in the gallbladder [14]. Such studies would be of relevance, given that ezetimibe could potentially not only inhibit NPC1L1 in the intestine and in hepatocytes but also in gallbladder epithelial cells, promoting gallstone formation, which contrasts with the clinical experience, since the drug is apparently associated with a decreased prevalence of cholesterol gallstone formation [14]. Since gallstone formation is a complex process, this observation could reflect the modulation of a number of important factors, in particular the substantial impairment of biliary cholesterol secretion by hepatocytes proximal of the gallbladder [14].

The cellular cholesterol efflux transporter ABCA1 is expressed on the gallbladder epithelial basolateral membrane [4]. Hepatic (70 \%) and intestinal ABCA1 (30\%) contribute significantly to the plasma levels of HDL cholesterol, whereas macrophage ABCA1 protects against the development of atherosclerosis [4]. Acceptors for cholesterol effluxed by ABCA1 in plasma are primarily small poorly lipidated discoidal HDL particles [4]. Interestingly, Yoon et al. [6] reported that the expression of ABCA1 was increased in gallbladders from patients compared to controls. This finding is surprising given that macrophage ABCA1 is significantly downregulated in response to proinflammatory stimuli $[15,16]$. However, increased basolateral ABCA1 expression could translate into increased cholesterol efflux from gallbladder epithelial cells as a mechanism to prevent cellular cholesterol overload as reported previously [17]. In the isolated normal human perfused gallbladder, addition of HDL to the arterial perfusate efficiently mobilizes cholesterol from the gallbladder [18]. However, gallbladder epithelial cells express and secrete apolipoprotein (apo)A-I and apoE [19]. The expression of apoA-I suggests the possibility that gallbladder epithelial cells export cholesterol in the form of HDL particles, potentially representing a novel cell type in addition to hepatocytes and enterocytes that can form HDL. In macrophages, apoE facilitates cellular cholesterol efflux [4]. Thereby, apoE expression in gallbladder epithelial cells might represent an additional factor facilitating to free these cells from excess cholesterol accumulation and related toxicity. Another relevant cholesterol export protein is ABCG1 mediating efflux towards larger HDL particles [4]. However, it is currently unclear if this transporter is expressed in gallbladder epithelial cells. Also unclear is how the presence of macrophage foam cells close to the basolateral side of gallbladder epithelial cells noted histologically in material from gallstone patients by Yoon et al. [6] should be interpreted. More work will be required to delineate how these macrophages obtain their lipid cargo and what the functional relationship with gallbladder epithelial cells is in cholesterol metabolism.

The impact of conditions such as inflammation or diabetes, which increase risk for gallstone development [2], should be further explored. Moreover, the transcriptional regulation of cholesterol transporters by bile acids either through farnesoid $\mathrm{X}$ receptor (FXR), fibroblast growth factor (FGF) receptors or the G-protein coupled receptor TGR5 (GPBAR1) would seem meaningful. Such data are relevant especially given the recent interest in FXR or 
TGR5 agonists for the treatment of metabolic disease [20]. The tissue expressing the highest levels of TGR5 is the gallbladder; interestingly, TGR5 knockout mice are protected from cholesterol gallstone development [21]. Therefore, treatment strategies using TGR5 agonists might be associated with an increased incidence of cholesterol gallstones.

In summary, the work by Yoon et al. [6] represents an interesting addition to the study of the active participation of the gallbladder in cholesterol metabolism, a topic that has not reached attention commensurate with its clinical importance. Future studies should aim to provide a more complete functional picture of the components of cholesterol metabolism expressed in gallbladder. Results from such studies would not only help to decide if the primary culprit for gallstone disease is the hepatocyte or the gallbladder epithelial cell, but would also provide the context for novel therapeutic approaches against cholesterol gallstone disease.

Acknowledgments This work was supported by a grant from the Netherlands Organization for Scientific Research (VIDI Grant 917-56-358, to UJFT).

\section{References}

1. Dikkers A, Tietge UJ. Biliary cholesterol secretion: more than a simple ABC. World J Gastroenterol. 2010;16:5936-5945.

2. Portincasa P, Ciaula AD, Bonfrate L, Wang DQ. Therapy of gallstone disease: what it was, what it is, what it will be. World $J$ Gastrointest Pharmacol Ther. 2012;3:7-20.

3. Nijstad N, Gautier T, Briand F, Rader DJ, Tietge UJ. Biliary sterol secretion is required for functional in vivo reverse cholesterol transport in mice. Gastroenterology. 2011;140: 1043-1051.

4. Annema W, Tietge UJ. Regulation of reverse cholesterol transport - a comprehensive appraisal of available animal studies. Nutr Metab (Lond). 2012;9:25.

5. Neiderhiser DH, Harmon CK, Roth HP. Absorption of cholesterol by the gallbladder. J Lipid Res. 1976;17:117-124.

6. Yoon JH, Choi HS, Jun DW et al. ATP-binding cassette sterol transporters are differentially expressed in normal and diseased human gallbladder. Dig Dis Sci. 2012. (Epub ahead of print). doi: 10.1007/s10620-012-2481-0.
7. Tauscher A, Kuver R. ABCG5 and ABCG8 are expressed in gallbladder epithelial cells. Biochem Biophys Res Commun. 2003;307:1021-1028.

8. Miquel JF, Moreno M, Amigo L, et al. Expression and regulation of scavenger receptor class B type I (SR-BI) in gall bladder epithelium. Gut. 2003;52:1017-1024.

9. Erranz B, Miquel JF, Argraves WS, Barth JL, Pimentel F, Marzolo MP. Megalin and cubilin expression in gallbladder epithelium and regulation by bile acids. J Lipid Res. 2004;45: $2185-2198$.

10. Wittenburg H, Lyons MA, Li R, Churchill GA, Carey MC, Paigen B. FXR and ABCG5/ABCG8 as determinants of cholesterol gallstone formation from quantitative trait locus mapping in mice. Gastroenterology. 2003;125:868-881.

11. von Kampen O, Buch S, Nothnagel $\mathrm{M}$ et al. Genetic and functional identification of the likely causative variant for cholesterol gallstone disease at the ABCG5/8 lithogenic locus. Hepatology. 2012. (Epub ahead of print). doi:10.1002/hep.26009.

12. Corradini SG, Elisei W, Giovannelli L, et al. Impaired human gallbladder lipid absorption in cholesterol gallstone disease and its effect on cholesterol solubility in bile. Gastroenterology. 2000; 118:912-920.

13. Hayashi A, Lee SP. Bidirectional transport of cholesterol between gallbladder epithelial cells and model bile. Am J Physiol. 1996; 271:G410-G414.

14. Pramfalk C, Jiang ZY, Parini P. Hepatic Niemann-Pick C1-like 1. Curr Opin Lipidol. 2011;22:225-230.

15. Khovidhunkit W, Moser AH, Shigenaga JK, Grunfeld C, Feingold KR. Endotoxin down-regulates ABCG5 and ABCG8 in mouse liver and ABCA1 and ABCG1 in J774 murine macrophages: differential role of LXR. J Lipid Res. 2003;44:1728-1736.

16. Maitra U, Li L. Molecular mechanisms responsible for the reduced expression of cholesterol transporters from macrophages by lowdose endotoxin. Arterioscler Thromb Vasc Biol. 2013;33:24-33.

17. Lee J, Shirk A, Oram JF, Lee SP, Kuver R. Polarized cholesterol and phospholipid efflux in cultured gall-bladder epithelial cells: evidence for an ABCA1-mediated pathway. Biochem J. 2002;364: 475-484.

18. Della Guardia P, Grossi A, Elisei W, et al. Plasma lipoproteins affect rate of cholesterol absorbed from bile by gallbladder: preliminary data. Ital J Gastroenterol Hepatol. 1999;31:587-592.

19. Lee J, Tauscher A, Seo DW, Oram JF, Kuver R. Cultured gallbladder epithelial cells synthesize apolipoproteins A-I and E. Am J Physiol Gastrointest Liver Physiol. 2003;285:G630-G641.

20. Porez G, Prawitt J, Gross B, Staels B. Bile acid receptors as targets for the treatment of dyslipidemia and cardiovascular disease. J Lipid Res. 2012;53:1723-1737.

21. Vassileva G, Golovko A, Markowitz L, et al. Targeted deletion of Gpbar1 protects mice from cholesterol gallstone formation. Biochem J. 2006;398:423-430. 